\title{
Incidencia del género y la edad sobre la calidad, satisfacción y valor percibido de los usuarios de actividades deportivas organizadas Incidence of gender and age on the quality, satisfaction and perceived value of users of organized sports activities \\ *Román Nuviala N uviala, **Raquel Pérez O rdás, *Guillermo M orán Gámez, **David Falcón Miguel * Universidad de Cádiz (España), ** U niversidad de Zaragoza (España)
}

Resumen. Los gestores deportivos buscan conocer las percepciones e implantar programas que garanticen la satisfacción y continuidad de los clientes, debido al incremento de práctica de actividad física y de la competitividad de los servicios deportivos. O bjetivo: constatar la existencia de diferencias en el modelo que relaciona calidad, satisfacción y valor percibido en función del género y la edad de los usuarios de actividades deportivas organizadas. Método: 1.654 personas (44.9 \% mujeres), con una edad media de $32.52 \pm 14.70$ años participaron en el estudio. El instrumento utilizado fue el cuestionario EPO D 2 y como pruebas estadísticas se real izaron anál isis exploratorios, correlaciones, alfadeC ronbach, AVE, FC, invariancias factorialesy coeficientes de regresión. Resultados: Lavaloración de la actividad fue positiva, siendo la satisfacción la percepción más puntuada, seguida por val or y calidad. Las mujeres valoraron mejor el servicio, encontrándose diferencias significativas por género en la val oración de calidad, y diferencias significativas en función de la edad y sexo en la evaluación de valor. La calidad tiene relación directay significativa con el valor, siendo ambos conceptos un antecedentedela satisfacción. Conclusiones: el usuario de actividades deportivas organizadas tiene una percepción muy positiva del ser vicio. Las mujeres valoran en mejor medida la calidad y el val or. A menor edad, menor estimación del valor. Los resultados del estudio justifican el establecimiento de estrategias de gestión para la mejora de la calidad y val or percibido con el fin de satisfacer y fidelizar al cliente.

Palabras clave: percepciones, gestor, servicio deportivo, estrategias, fidelización.

Abstract. Sports managers try to understand customer perceptions and to implement programmes that guarantee clients satisfaction and continuity, because of the increase in the practice of physical activity and the competitiveness of sports services. 0 bjective: to deter mine the existence of differences in the model that rel ates qual ity, satiffaction and perceived val ue according to the gender and age of the users of organized sports activities. M ethod: 1.654 people (44. $9 \%$ women), with a mean age of $32.52 \pm 14.70$ years participated in the study. The instrument used was the EPO D2 questionnaire and exploratory tests, correlations, Cronbach's alpha, AVE, composite reliability, factorial invariances and regression coefficients were used as statistical tests. Results: the activity assessment was positive, with satisfaction as the highest rated perception, followed by value and qual ity. Women rated the service better, finding significant differences by gender in the assessment of quality, and significant differences according to age and sex in the assessment of val ue. Q ual ity has adirect and significant relationship with val ue, both concepts being an antecedent of satisfaction. Conclusions: the user of organized sports activities has a very positive perception of the service. Women rate quality and val ue more highly. The younger the age, the lower the value assessment. The results of the study justify the establishment of management strategies for the improvement of qual ity and perceived value in order to satisfy customers and build customer loyalty.

Keywords: perceptions, manager, sport service, strategies, loyalty.

\section{Introducción}

En los últimos años, el interés por la actividad física y la práctica deportiva ha aumentado (IHRSA, 2019), debido fundamentalmentealoscuantiosos beneficiosque conlleva su práctica regular y adecuada, sobre diferentes dimensiones de la salud de la persona que la realiza (Castañeda, Campos, \& Castillo, 2016). En consecuen-

Fecha recepción: 25-09-20. Fecha de aceptación: 06-03-21 Román Nuviala Nuviala

roman.nuviala@gm.uca.es cia, el número de servicios relacionados con el bienestar, la actividad física o el deporte ha crecido, con el objetivo de responder a dicha demanda (León, García, \& Burillo, 2020).

El incremento de estos modelos de servicios y su competitividad ha repercutido en que los gestores deportivos se preocupen más conscientemente por mejorar su oferta de servicios (Zamorano \& García, 2018). Este hecho ha extendido la investigación en las variables que podrían repercutir en la fidelidad o continuidad del cliente con el servicio (García-Fernández, Bernal-García, Fernández-Gavira, \&Vélez-Colon, 2014) 
como puedan ser la percepción de la calidad, la satisfacción y el valor percibido.

Numerosas investigaciones corroboran que existe una fuerte correlación entre la calidad percibida y la satisfacción con el servicio deportivo, apoyando la idea de que la satisfacción del cliente resulta de la calidad de servicio percibida (Álvarez, González, Río-Rama, de la Cruz, \& Durán, 2019; Zopiatis, Theocharous, Constanti, \& Tjiapouras, 2017; Yildiz \& Duyan, 2019; Castillo, O netti, \& Chinchilla, 2019) y que la calidad del servicio antecede directamente al valor percibido (Álvarez et al., 2019; Moreno, Prado-Gascó, Hervás, Núñez-Pomar, \& Sanz, 2015; N uviala, Grao-Cruces, Fernández-O zcorta, \& Nuviala, 2015). En el caso de la relación entre valor percibido y satisfacción, los diferentes estudios en el ámbito deportivo también afirman que la satisfacción es una consecuencia del valor percibido (Theodorakis, Howat, Ko, \& Avourdiadou, 2014; N uviala, Aznar-Cebamanos, Grao-Cruces, \& Nuviala, 2020; M olina-García\& González-García, 2018; Chiu, Won, \& Bae, 2019; García, Alguacil, \& M olina, 2020).

En el campo específico de los servicios deportivos diferentes estudios también han revelado la relación positiva y conjunta entre calidad percibida, valor percibido y satisfacción, con la continuidad de los usuarios en estos servicios, resultando que cuanto más positivas sean las percepciones de los usuarios, más posibilidades existirán de que estos continúen utilizando los servicios deportivos (M urray \& Howat, 2002; Theodorakis et al., 2014; H aro, Pérez-O rdás, Leyton, Caamaño, \& N uviala, 2018a). A partir del conocimiento de las relaciones entre estos constructos, se pueden establecer model os que ofrezcan una mejor comprensión de las actitudes de los clientes al disfrutar posteriormente del servicio (Pra do-Gascó, Calabuig, Crespo, \& Núñez-Pomar, 2018). De este modo, el gestor deportivo podrá dar respuestas a sus necesidades, objetivos y expectativas, consiguiendo su fidelización (Bernal-García, GarcíaFernández, Grao-Cruces, Teva-Villén, \& N uviala, 2018; García-Fernández, Gálvez-Ruíz, Pitts, Vélez-Colón, \& Bernal-García, 2018; M ontesinos, Vegara, Morales, López, López, \& Angosto, 2018; Haro, Pérez-O rdás, Grao-Cruces, Nuviala, \& Nuviala, 2018b).

Por todo ello, a la hora de implementar cualquier programa de gestión que tenga como objetivo incrementar la eficacia y eficiencia del servicio, así como la satisfacción del cliente y su continuidad, se deberá llevar a cabo una serie de mediciones que proporcionen información sobre las percepciones de los usuarios
(Grijalva, Cevallos, \& Jiménez, 2020; Nuviala, TamayoFajardo, Gómez, Nuviala, \& Torres, 2017). Esto puede constituir una ventaja frente a la competencia, facilitando el logro de un posicionamiento estratégico adecua do, resultando útil desde el punto de vista de la gestión y favoreciendo la toma de decisiones del gestor deportivo (Haro, et al., 2018a) contribuyendo a incrementar la satisfacción, la fidelización al servicio y la práctica deportiva.

Cada vez son más los trabajos que evalúan las percepciones de los usuarios de servicios deportivos (Calabuig, Q uintanilla, \& M undina, 2008) y los autores que crean instrumentos específicos para medir las percepciones de los usuarios y adaptarse a las necesidades específicas de cada uno de los servicios deportivos (Ramírez, Lorenzo, \& López, 2017).

Sin embargo, a pesar de conocerse que variables demográficas como el género, la edad, los ingresos y el nivel de estudios tienen gran incidencia en las expecta tivas, las percepciones, la satisfacción y las intenciones futuras delosusuarios(Vergara, Q uesada, \& M aza, 2017) son escasos los estudios que relacionan este tipo de va riables. Tampoco son numerosos los trabajos que integran la relación entre los tres constructos (calidad, sa tisfacción y valor percibido) con variables sociodemográficas (Pérez-O rdás, Aznar-Cebamanos, N uviala, \& Nuviala, 2019) en el campo deportivo. Principalmente se encuentran estudios que asocian calidad y/ 0 satisfacción con género y/ o edad, como los de García-Fernández et al. (2014) sobre la percepción de calidad según edad y sexo en clientes de centros de fitness low cost; M olina García y González-García (2018) que relacionan calidad y satisfacción con edad y sexo en escuelas náuticas; Castillo et al. (2019) que conectan las variables de edad y sexo con la calidad en centros deportivos municipales de Málaga; León et al. (2020) que establecieron una relación entre construcciones como edad y sexo y la calidad y satisfacción percibida en 25 centros de fitness de gestión privada de la Comunidad de Madrid; Berber y Mollaoðullar1 (2020) en el que relacionaron la edad y el género con la calidad y satisfacción percibida del servicio recibido en participantes de los programas deportivos de la Dirección Provincial de J uventud y Deportes de Izmir; Calesco y Both (2020) en el que analizan la calidad del servicio prestado por el monitor degimnasiosubicadosen la ciudad de Londrina Paraná teniendo en cuenta la edad y el sexo; García, Carcelén, Sánchez y Suárez (2017) que estudian la satisfacción según la edad y sexo de clientes externos en una piscina cubierta u otras que analizan la calidad percibida 
general de clientes en centros fitness (Zamorano \& García, 2018). Estudios que relacionen calidad y valor con edad y sexo también son limitados, como el de García-Fernández, Grimaldi, Gómez y Bernal (2016), que investigaron esta relación en centros fitness lowcost.

Las investigaciones recientes que relacionen calidad, satisfacción y valor percibido con variables sociodemográficasen un solo trabajo, siguen siendo también reducidas, pudiéndose enumerar las de Pérez-0 rdás et al. (2019) sobre actividades deportivas extracurriculares; García, Gómez y Tafalla (2019) sobre el perfil del usuario de centros deportivos privados; Haro et al. (2018b) sobre la satisfacción de usuarias de centros deportivos unisex y exclusivos para mujeres 0 García et al. (2017) en un análisis sobre usuarios de una Piscina M unicipal. Este hecho y basándonos en los anteriores autores justifica la pertinencia de relacionar estos tres constructos con las variables sociodemográficas de edad y género, lo que ayudará a establecer estrategias de gestión para la mejora de los servicios relacionados con la actividad deportiva (Haro, et al., 2018a).

El presente manuscrito desarrolla las secciones de material y métodos incluyendo descripción de los participantes, instrumento, procedimiento, análisis de da tos; resultados, discusión, conclusiones y referencias. El objetivo de este trabajo es constatar la existencia de diferencias en el modelo que relaciona calidad, satisfacción y valor percibido en función de género y edad de usuarios de actividades deportivas organizadas, lo que plantea tres hipótesis principales: la existencia de diferencias en el modelo de relación de calidad, satisfacción y valor percibido en función del género $(\mathrm{H} 1)$ y en función de la edad (H2), y señalar a la calidad del servicio como un antecedente del valor percibido y a ambos como precursores de la satisfacción (H3).

\section{Material y M étodo}

\section{Participantes}

La población de este estudio fue de 1.654 personas seleccionadas al azar (44.9\% mujeres; edad media de $32.52 \pm 14.70$ años) que realizan actividades físicas organizadas en 56 centros deportivos públicos y privados de España. El tiempo medio de permanencia en la organización erade $5.82 \pm 6.94$ años. El $48.3 \%$ poseían estudios superiores y el $66.7 \%$ del total realizaban práctica de actividad físico-deportiva 3 veces o menos a la sema na.

\section{Instrumento}

Para la realización de este trabajo se utilizó el cuestionario de percepción de organizaciones deportivas (EPO D2) (Nuviala, Grao-Cruces, Teva, Pérez-Turpin, Pérez-O rdás, \& Tamayo, 2013). El instrumento está conformado por 25 ítems que miden, utilizando una escala de cinco puntos que va desde muy en desacuerdo (1), a muy de acuerdo (5), los constructos de calidad, valor y satisfacción. La calidad es valorada mediante 20 ítems, cuya fiabilidad medida tras el trabajo de campo con Alpha de Cronbach fue de .934. La satisfacción es medida mediante 4 ítems, y la fiabilidad medida con Alpha de Cronbach fue de .934. El valor es medido mediante 1 ítem (Tabla 1).

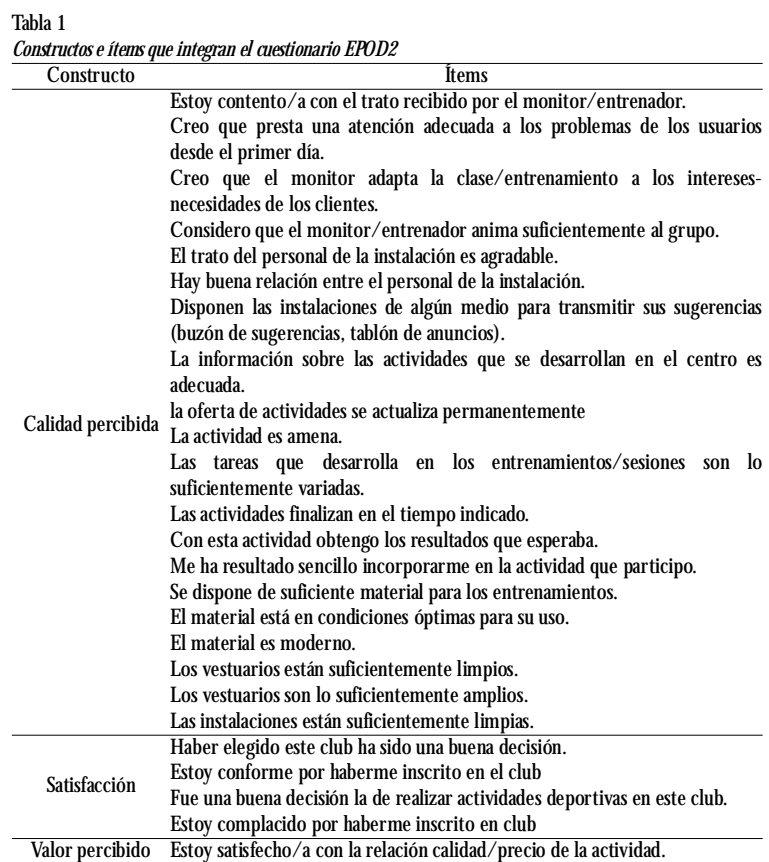

A las escalas se le añadieron varias preguntas sociodemográficas como edad, género, estudios, frecuencia de práctica semana, tiempo medio por sesión, tiempo que llevan practicando y tipo de actividad que realiza.

\section{Procedimiento}

Los encuestados recibieron instrucciones para valorar el grado de acuerdo con cada ítem de forma previa a la contestación del cuestionario. El trabajo cumplió con los más altos estándares de seguridad y ética siendo aprobado por la comisión ética de la unta deAndalucía. Se informó a los responsables de las organizaciones que participaron en el estudio de los objetivos y metas de la investigación, que se llevó a cabo tras la obtención de la aprobación dela organización. El diseño del estudio consideró todo el marco legal español que regula la protec- 
ción de datos personales de acuerdo con la Ley O rgánica 15/ 1999. También se tuvieron en cuenta en todo momento los principios fundamental es establecidos en la Declaración de Helsinki (revisada en 2013, Brasil). Se obtuvo el consentimiento informado de todos los participantes en el estudio antes de realizar el trabajo de campo. Posteriormente, se utilizó el cuestionario autoadministrado en presencia de un investigador. El tiempo dedicado a completar los cuestionarios fue de aproximadamente 10 minutos, dentro de la misma instalación.

\section{Análisis de los datos}

En un primer momento se realizaron una serie de pruebas exploratorias como son el cálculo de frecuencias, medias, desviación típica, asímetría y curtosis. Se calcularon las correlaciones entre los constructos que conforman la investigación y alfa de Cronbach, todo ello con el paquete estadístico SPSS V22. Los valores deAlpha de Cronbach estuvieron por encima del nivel recomendado de 0,70, lo cual indicó que la escala posee unabuena consistencia interna (Tabla 2). De igual forma se calculó la varianza media extraída (AVE) y la fiabilidad compuesta ( $\mathrm{FC}$ ). A continuación, se realizó un análisis multigrupo con el programaAM OS v.22. Este procedimiento permite verificar la invariabilidad de la estructura factorial del modelo sometido a estudio. El objetivo del análisis es mostrar si el modelo que relaciona la calidad, el valor y la satisfacción por el servicio recibido, es el mismo para los grupos de usuarios divididos en función del género y la edad. Lanormalidad multivariada de los datos fue determinada mediante el coeficiente Mardia, por lo que, posteriormente, se realizó el análisis con el método de máxima verosimilitud. Para eva luar la invariancia factorial, se siguió el procedimiento mediante el cual se debe verificar el ajuste del modelo en diferentes modelos. El ajuste de cada modelo se eva luó mediante el examen de varios índices: Comparative Fit Index (CFI), Root M ean Square Residual (RMR), Root Mean Square Error of Approximation (RMSEA), Goodness-of Fit Index (GFI) y Expected Cross-Validation Index (ECVI), se utilizaron como estadísticos de ajuste. Asimismo, se utilizó el valor de la razón de Chi-cuadra do (CM IN) y el valor de Chi-cuadrado partido por los grados de libertad (CM IN/ DF). Finalmente, se calcu-

\footnotetext{
Tabla 2

Media y desviación típica. Anova y nivel de significación. Correlación entre constructos, Alfa de Cronbach en la diagonal (entre paréntesis), AVE y FC.

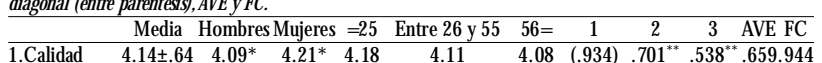

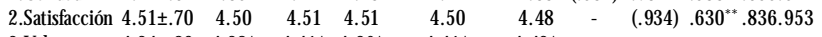
3.Valor $\quad 4.34 \pm 89 \quad 4.28^{*} \quad 4.41 * 4.26^{*} \quad 4.41 * \quad 4.43^{*}$ 3. Valor $\quad 4.34 \pm .01 ; * p<.05$
}

laron los coeficientes de regresión estandarizados para las relaciones en el modelo en función del género y edad de los usuarios de los centros de fitness.

\section{Resultados}

Como se puede ver en la Tabla 2, la satisfacción ha obtenido el valor medio más alto y la calidad obtiene una media inferior al resto de constructos analizados. Se han encontrado diferencias significativas entre hombres y mujeres en la valoración de la calidad y en el valor, siendo las mujeres las que valoran en mejor medida el servicio. De igual forma se observa la existencia de diferencias significativas en el valor en función del grupo de edad (Tabla 2).

Se eval uó la consistencia interna para cada construcción de investigación. Los resultados de la prueba de Composite Reliability (FC) revelaron que todos los va lores fueron mayores que 0.6. El cálculo de la Average Variance Extracted (AVE) respalda la validez convergente ya que los valores resultantes AVE de los constructos de investigación estaban cerca o por encima del valor recomendado de 0.5 (Tabla 2). La validez discriminante de los datos se constató mediante el cálculo de la matriz de correlaciones entre los factores. Como puede observarse existió correlación significativa y positiva entre los factores que componen el estudio (Tabla 3).

\begin{tabular}{|c|c|c|c|c|c|c|c|c|}
\hline \multicolumn{9}{|c|}{ 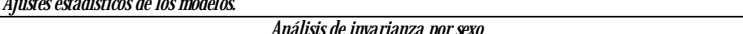 } \\
\hline Modelo & CMIN & DF & CMIN/DF & $\mathrm{CFl}$ & RMSEA & RMR & $\mathrm{GFI}$ & ECVI \\
\hline 0 & 547.272 & 257 & 2.129 & .963 & .050 & .045 & .909 & 1.505 \\
\hline 1 & 691.768 & 514 & 1.346 & .977 & .038 & .049 & .887 & .849 \\
\hline 2 & 699.025 & 537 & 1.302 & .979 & .038 & .051 & .886 & .834 \\
\hline 3 & 702.324 & 539 & 1.303 & .979 & .037 & .053 & .886 & .822 \\
\hline 4 & 705.034 & 541 & 1.303 & .979 & .038 & .056 & .886 & .829 \\
\hline 5 & 758.052 & 582 & 1.302 & .977 & .048 & .058 & .878 & .940 \\
\hline \multicolumn{9}{|c|}{ Análisis de invarianza por edad } \\
\hline Modelo & CMIN & DF & CMIN/DF & $\mathrm{CFl}$ & RMSEA & RMR & $\mathrm{GFI}$ & ECVI \\
\hline 0 & 547.272 & 257 & 2.129 & .963 & .050 & .045 & .909 & 1.505 \\
\hline 1 & 971.200 & 839 & 1.158 & .983 & .019 & .107 & .853 & 2.744 \\
\hline 2 & 996.941 & 862 & 1.157 & .983 & .019 & .111 & .848 & 2.700 \\
\hline 3 & 1000.936 & 864 & 1.158 & .982 & .019 & .111 & .848 & 2.700 \\
\hline 4 & 1001.069 & 866 & 1.156 & .983 & .019 & .110 & .848 & 2.691 \\
\hline 5 & 1134.237 & 907 & 1.251 & .971 & .024 & .110 & .828 & 2.804 \\
\hline \multicolumn{9}{|c|}{$\begin{array}{l}\text { Nota. Modelo 0, resultante del A nálisis Factorial Confirmatorio; Modelo } 1 \text {, no tiene } \\
\text { restricciones de ningún tipo; Modelo } 2 \text {, tiene restricciones en el peso de medida; Modelo } 3 \text {, } \\
\text { tiene restringidos los pesos de medida y covarianzas; Modelo 4, tiene restricciones en los pesos } \\
\text { de medida, covarianzas y residuos de medida; Modelo 5, tiene restricciones en los pesos de } \\
\text { medida, covarianzas y residuos de mediday estructurales. }\end{array}$} \\
\hline
\end{tabular}

Se procedió en primer lugar a conocer el valor del coeficiente multivariado de Mardia que obtuvo un va lor de 380.938. Se utilizó para el análisis el método de máxima verosimilitud. La validez de la estructura factorial del modelo que relaciona calidad, valor y sa tisfacción es correcta puesto que los índices de bondad de ajuste del Análisis Factorial Confirmatorio (AFC) son excelentes (Tabla 3; modelo 0). El modelo 
muestra relaciones entre calidad, valor y satiffacción (Tabla 5).

Comprobado que el modelo es correcto y para comparar el modelo en función del género de los usuarios, es necesario realizar pruebas de invariancia factorial. $\mathrm{Si}$ se tiene en cuenta la diferencia en CM IN/ DF entre el modelo sin restricciones (modelo 1 ) con los modelos con restricciones modelos 2, 3, 4 y 5, no se han observado diferencias (Tabla 4). U sando como correcto el modelo 2, la tabla 4 muestra la no existencia de diferencias con el resto de modelos. El modelo 3, tampoco presenta diferencias con el modelo 4 y 5, así como no existen diferencias entre el modelo 4 y 5 . Las diferencias en $\div 2$ permiten aceptar la hipótesis de invarianza. Si además se observa el valor de CFI en los modelos, se puede apreciar que todos ellos presentan valores muy similares, con una diferencia entre ellos menor a - .01 . Todo ello sugiere la invarianza factorial del modelo en función del género (Tabla 3).

Para comparar el modelo en función de la edad de los usuarios, es necesario realizar pruebas de invariancia factorial. Si se tiene en cuenta la diferencia en CM IN/ DF entre el modelo sin restricciones (modelo 1) con losmodelos con restricciones modelos 2, 3 y 4 no se han observado diferencias. Si existen diferencias entre el modelo 1 y 5 . U sando como correcto el modelo 2, la tabla 4 muestra la no existencia de diferencias con los modelos 3 y 4 . Si aparecen diferencias significativas con el modelo 5. El modelo 3, presenta diferencias con el modelo 5, así como existen diferencias entre el modelo 4 y 5 (Tabla 4). Las diferencias en $\div 2$ no permiten aceptar la hipótesis de invarianza, pero el resto de índices contradicen esta conclusión. Al observar el valor de CFI en los modelos, se puede apreciar que todos ellos, exceptuando el valor del modelo 5, presentan valores muy similares, con una diferencia entre ellos menor a,01. Las variaciones en RM SEA son inexistente a diferencia del modelo 5 que es de.05. De igual forma, al observar el índice ECVI, puede advertirse que las diferencias en los ajustes son mínimos, excepto el modelo 5, lo cual indica que los diferentes modelos presentan valores muy similares. Todo ello sugiere la invarianza factorial del modelo en función de la edad (Tabla 3).

El modelo 1 presenta un conjunto de valores correctos en todos los índices. Además, observando los valores de este modelo, en relación al resto de modelos examinados, tanto para el género como para la edad, presenta valores muy similares al resto y siempre positivos. Por lo tanto, este modelo se considera bueno para realizar la comparación en función del género y la edad.
Tabla 4

Comparación de las condiciones de invarianza

\begin{tabular}{|c|c|c|c|c|c|c|c|}
\hline & \multicolumn{5}{|c|}{ Invarianza por sexo } & \multicolumn{2}{|c|}{ Invarianza por edad } \\
\hline & Modelo & 0 Dif.DF & Dif.CMIN & $p$ & Dif. DF & Dif.CMIN & $p$ \\
\hline \multirow{4}{*}{$\begin{array}{l}\text { Asumiendo que el } \\
\text { modelo } 1 \text { es correcto }\end{array}$} & 2 & 23 & 7.257 & .999 & 23 & 25.741 & .313 \\
\hline & 3 & 25 & 10.555 & .995 & 25 & 29.736 & .234 \\
\hline & 4 & 27 & 13.266 & .987 & 27 & 29.869 & .320 \\
\hline & 5 & 68 & 66.283 & .536 & 68 & 163.038 & .000 \\
\hline \multirow{3}{*}{$\begin{array}{l}\text { Asumiendo que el } \\
\text { modelo } 2 \text { es correcto }\end{array}$} & 3 & 2 & 3.299 & .192 & 2 & 3.995 & .136 \\
\hline & 4 & 4 & 6.009 & .198 & 4 & 4.128 & .389 \\
\hline & 5 & 45 & 59.027 & .078 & 45 & 137.296 & .000 \\
\hline \multirow{2}{*}{$\begin{array}{l}\text { Asumiendo que el } \\
\text { modelo } 3 \text { es correcto }\end{array}$} & 4 & 2 & 2.711 & .258 & 2 & .133 & .936 \\
\hline & 5 & 43 & 55.728 & .092 & 43 & 133.302 & .000 \\
\hline $\begin{array}{l}\text { Asumiendo que el } \\
\text { modelo } 4 \text { es correcto }\end{array}$ & 5 & 41 & 53.017 & .099 & 41 & 133.169 & .000 \\
\hline \multicolumn{8}{|c|}{$\begin{array}{l}\text { Nota. Modelo 1, no tiene restricciones de ningún tipo; Modelo 2, tiene restricciones en el peso } \\
\text { de medida; M odelo } 3 \text {, tiene restringidos los pesos de medida y covarianzas; Modelo 4, tiene } \\
\text { restricciones en los pesos de medida, covarianzas y residuos de medida; Modelo } 5 \text {, tiene } \\
\text { restricciones en los pesos de medida, covarianzas y residuos de mediday estructurales. } \\
\text { Dif. CMIN, diferencia entre modelos de chi-cuadrado; Dif. DF, diferencia entre modelos de } \\
\text { grados de libertad; p, nivel de significación entre modelos. }\end{array}$} \\
\hline
\end{tabular}

En laTabla 5, los resultados muestran que la cal idad tiene relación directa y significativa con el valor, en la población total y en todos los grupos. Son las mujeres y los usuarios del grupo con edades comprendidas entre 26 y 55 años, quienes presentan unos valores beta más elevados. La calidad es un antecedente de la satiffacción en todos los grupos. Es en el grupo de personas con edades comprendidas entre 26 y 55 años, donde aparece el valor beta más bajo. Finalmente, el valor es un precedente de la satisfacción. Destaca el valor beta para el grupo de edad de entre 26 y 55 años, que presenta el valor beta más elevado (Tabla 5).

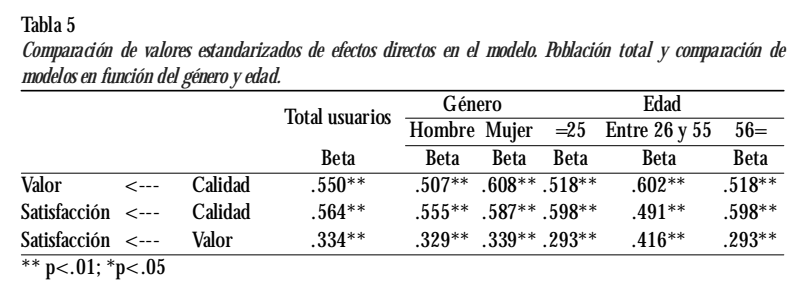

\section{Discusión}

El objetivo de este trabajo ha sido constatar la existencia de diferencias en el modelo que relaciona calidad, satisfacción y valor percibido en función del género y la edad de usuarios de servicios deportivos que realizan actividades físicas organizadas, siendo numerosas las investigaciones que estudian y val oran cual quiera de estos constructos en el entorno deportivo (Chiu et al. , 2019; Garcíaet al. , 2020; Barshan, Elahi, \&Aghaei, 2017; Días, Ferreira, Romao-Pereira, \& Fonseca, 2019) y las que los relacionan con diferentes tipos de variables como puedan ser la experiencia previa (M ontesinos et al., 2018), si la actividad es libre o guiada (Prado-Gascó et al., 2018), si se es responsable técnico o político del municipio que presta el servicio (Solanellas $\&$ Macías, 2019), la edad o el sexo (León et al. , 2020) o la influencia del bienestar subjetivo dentro de los programas que 
ofrecen los distintos servicios deportivos (GarcíaPascual, Silla-Merchán, Mundina, \& EscamillaFajardo, 2016). El análisis de estos factores, principalmente las variables sociodemográficas (Vergara et al., 2017), ha puesto de manifiesto la importancia de una percepción positiva del servicio debido a su relación directa con la fidelidad de losclientes(Theodorakiset al. , 2014), siendo su análisis crucial en el sector del fitness debido a los al tos niveles de abandono existentes (García-Fernández, Gavira, \& García, 2014). El conocimiento de los clientes y de sus juicios es una de las vías principales para la mejora de la gestión y prestación de estos servicios y de la satisfacción de los usuarios, lo que se traduce en un incremento de la lealtad y fidelización de los mismos (N uviala et al., 2017; Calesco \& Both, 2020).

Esta investigación revela una buena valoración de la calidad, el valor percibido y la satisfacción de los usua rios de actividades físicas organizadas, siendo la satisfacción el constructo con mejor valoración, seguido por el valor y la calidad percibida, corroborando los hallazgos de estudios anteriores (García et al., 2017; García et al.,2019; M olina, 2016) en diferentes servicios y centros deportivos.

El hecho de que lasatisfacción sea el constructo mejor valorado seguido por el valor percibido, podría explicarse porque el valor percibido, variable resultante de una evaluación combinada de la percepción de los beneficios y sacrificios (Boksberger \& M elsen, 2011) ha sido señalado como un mediador importante entre la calidad del servicio y la satisfacción de los usuarios (N uviala, Pérez-O rdás, 0 suna, Grao-Cruces, Nuviala, \& Jurado, 2012; Chiu et al., 2019) así como un predictor de la satisfacción del usuario (García et al., 2020) en el contexto de diferentes empresas de servicios (Brady, Knight, Cronin, Hult, \& Keillor, 2005) y también en la de servicios deportivos (M urray \& Howat, 2002). Diferentes resultados obtuvieron Montesinos et al. (2018) y Calabuig, Burillo, Crespo, M undinay Gallardo (2010), ya que en sus estudios examinan las percepciones de espectadores y participantes de competiciones deportivas, y el valor percibido, en relación a la calidad percibiday satisfacción general es el constructo que peor registro presenta. Esta apreciación puede ser debida a que el valor es un término de naturaleza dinámica, multidimensional, compuesto por elementos cognitivos y afectivos, de percepción subjetiva, con un carácter relativo y que puede variar entre personas y situaciones (Haro, 2018). En situaciones influenciadas por algo tan variable y emotivo como el resultado de una competición, puede resultar que la relación calidad/ precio de la actividad sea la percepción peor valorada.

La valoración de la calidad del servicio ha sido positiva, al obtener una puntuación media para el total de

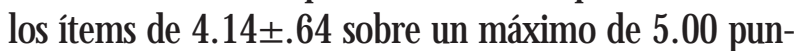
tos, encontrándose diferencias significativas entre hombres y mujeres. Resultados que son similares o superiores a los hallados por García-Fernández et al. (2016), Haro (2018), Montesinos et al. (2018), Castillo et al. (2019), Nicolás-López y Escaravajal-Rodríguez (2020) 0 Calesco y Both (2020).

El grupo de menores de 25 años fue el grupo que mayor valoración otorgó a la calidad percibida, aspecto que puede ser debido a su inexperiencia como usuarios de servicios deportivos y su menor conocimiento de aspectos tangibles del centro deportivo donde realiza el ejercicio. Cuanto mayor sea la edad del usuario, las probabilidades de haber acumulado más experiencias aumentan, incrementando el conocimiento de los temas organizacionales, la planificación de las acciones de la empresa y la relación con el cliente, transformando al usuario en una persona más exigente y crítica con el servicio (Q uintana, M olina, \& M olina, 2010; García, et al. , 2019; Calesco \& Both, 2020).

La satisfacción con los servicios deportivos examinados fue el término más valorado (4.51土.70), en línea con los resultados de otros estudios (Prado-G ascó et al., 2018; M ontesinos et al. , 2018; Garcíaet al., 2017; RosCastello, Calabuig, \& Gómez, 2018; Yildiz \& Duyan, 2019; A paricio, Elasri, \&Triadó, 2018). Esta calificación superior, puede deberse a las características específicas de la calidad como una opinión que dura en el tiempo, 0 del valor percibido como un término que está compuesto por elementoscognitivosy afectivos(Haro, 2018), mientras que la satisfacción es una opinión temporal que se refiere a un servicio específico (Nuviala et al., 2013; Kamolrat, Laat, \& M itas Rooij, 2019) y que depende en gran medida de losaspectosemocionales(Chiu et al., 2019; Kamolrat et al., 2019; M onroy, 2019). La valoración de la satisfacción supone una evaluación posterior al consumo y/ 0 al uso, lo que conlleva que es una percepción que podría cambiar en cada transacción y que es el resultado de procesos psicosociales cognitivos y afectivos. Los gestores de los servicios deportivos no pueden ignorar este aspecto, y deben tratar de relacionar la actividad física con el desarrollo y la mejora de los procesos afectivos y cognitivos entre sus usuarios (Pérez-0 rdás et al., 2019).

Respecto al valor percibido (relación calidad/ precio de la actividad), su valoración fue de 4.34 4.89 , ha llándose diferencias significativas $(p<.001)$ en función 
del sexo y del grupo de edad, al igual que el trabajo de García-Fernández et al. (2016). Coincidiendo con investigaciones anteriores (García-Fernández et al., 2016; García et al., 2019; Molina, 2016; Solís-Radilla, Hernández-Lobato, \& Villagómez-M éndez, 2016) fueron los mayores de 26 años los que hicieron una valoración superior de valor percibido. Por otro lado, los participantes menores de 25 años fueron los que otorgaron una menor valoración a este constructo, coincidiendo con Nicolás-López y Escaravajal-Rodríguez (2020) 0 Pérez-O rdás et al. (2019). Con estos resultados podemos decir que el aumento de la valoración del valor percibido en función de la edad se puede deber a que los adultos poseen un mayor poder adquisitivo, lo que provocaun aumento dela percepción calidad-precio (Elasri, Triadó \& A paricio, 2014), que están laboralmente estabilizados y centralizan en el disfrute personal las alternativas de selección de actividad y no en el ahorro (Q uintana et al., 2010), lo que les permite elegir el servicio en función de sus exigencias.

Independientemente del sexo de los encuestados, la valoración de los tres constructos fue muy positiva, ha llándose diferencias significativas entre hombres y mujeres en la valoración de la calidad y valor percibido. Las mujeres han valorado en mejor medida el servicio deportivo recibido, resultado similar a los obtenidos por García-Fernández et al. (2016); Castillo et al. (2019); M olina-García y González-García (2018), García et al. (2019) y Haro et al. (2018b). Estas evaluaciones pueden ser debidas a que las mujeres suelen otorgar preferencia a los esquemas de valoración de la cal idad subjetivos (cumplir sus expectativas, esforzarse por mejorar y comprometerse) y de un tinte más afectivo, se adhieren más a las actividades del grupo en fitness (ej., clases de aerobic, step, spinning) e incluso a algunas actividades más específicas (Campos, González, González, \& Martins, 2019). Esta predilección por parte de las usuarias se manifiesta en una mayor demanda de actividades grupales y organizadas, cuyo objetivo sería la mejora de la sensación de bienestar o socio-afectivo (0 rtega, Lourenço, González-Villora, \& Campos, 2021). Por el contrario, loshombres se muestran como un grupo más crítico (García et al., 2014; Calesco \& Both, 2020) ba sando sus juicios en dimensiones objetivas como puede ser el precio (Salvador, 2007).

Los resultados del estudio señalan que existe una relación directa y significativa entre la calidad y el valor percibido en todos los grupos del estudio, datos que están en consonancia con otros estudios que relacionan percepciones con variables sociodemográficas de edad y sexo (García et al., 2017; García-Fernández et al., 2016; Haro, 2018; Pérez-O rdás et al., 2019). Si comparamos los resultados con otros trabajos en los que no se hace mención al sexo y edad de los clientes, los hallazgos de este estudio mantienen la línea de los de investigaciones anteriores (N uviala et al. 2015; GarcíaFernández et al. , 2018; M ontesinos et al., 2018).

La calidad del servicio se ha confirmado como un antecedente de la satisfacción (Álvarez et al., 2019; Berber \& M ollaoðullar1 ,2020; Barshan et al., 2017; M olina-García\& González-García, 2018; GarcíaPascual et al. , 2016; Haro et al., 2018b) constatando que el valor percibido tiene relación directa con la satisfacción, actuando como predictor (Theodorakis et al. 2014; Molina-García \& González-García, 2018; GarcíaFernández et al., 2018; García et al., 2020; N uviala et al., 2020). La relación entre las tres variables estudia das reprodujo correctamente el modelo propuesto por M urray y H owat (2002) en usuarios de servicios deportivos que realizan actividades físicas organizadas.

Por tanto, si la calidad percibida es antecesora del valor, y la satiffacción es resultado del valor y la calidad percibida (GarcíaFernández et al. , 2018; Nicolás-López \& Escaravajal-Rodríguez, 2020; $H$ aro et al., 2018b; M ontesinosetal., 2018; Pérez-O rdáset al., 2019), cuanto más positivas sean las percepciones del usuario, más posibilidades habrá de que siga participando en los servicios deportivos.

Como limitaciones de este estudio encontramos la omisión de variables que podrían haber definido más el perfil sociodemográfico de los usuarios, especialmente su nivel socioeconómico. Deben realizarse más estudios en el campo de los servicios deportivos diferenciando género y edades, centros públicos o privados, para buscar perfiles de gestión deportiva más apropiada para la población objeto. 0 tra limitación de este estudio es que los cuestionarios solo están val idados en espa ñol, lo cual es una desventaja cuando se habla de ellos en otros entornos.

La investigación futura podría abordar los efectos del COVID-19 sobre lafidelidad y percepciones de usua rios de actividades deportivas organizadas, ampliar tanto la muestra poblacional como la variedad de factores considerados, para así tener en cuenta los diferentes tipos de usuarios y contribuir sustancialmente a la literatura existente sobre el desarrollo teórico de este modelo, además de su replicabilidad en diferentes contextos sociodemográficos. Sería interesante validarlos en otrosidiomasy poder extender esta investigación aotros países. 


\section{Conclusiones}

Como principales conclusiones de este trabajo podemos indicar que el usuario de actividades deportivas organizadas tiene una percepción muy positiva del servicio recibido, siendo la satisfacción el elemento más valorado, seguido por el valor y la calidad percibida. Son los menores de 25 años y las mujeres quienes valoran en mejor medida la calidad percibida del servicio. En cuanto a la evaluación del valor percibido, fueron mujeres y mayores de 26 años quienes lo valoraron con mejor puntuación.

Los resultados también nos indican la relación funcional que señala a la calidad del servicio como antecedente del valor percibido y a ambos, como precursores de la satisfacción corroborando la tercera hipótesis del estudio. Se ha podido observar que existe una homogeneidad en los resultados, si bien, aparecen diferencias en los valores beta estandarizados en las dos variables sociodemográficas: edad y sexo, aspecto que nos permite ratificar las dos primeras hipótesis.

Desde una perspectiva práctica, la correcta gestión de la calidad percibida es clave para la gestión de actividadesfísicas organizadas, por su influencia en la percepción del valor y satisfacción del cliente. Demostrada la influencia positiva y conjunta que estos tres constructos (calidad, satiffacción y valor) tienen sobre la conducta de los usuarios y en la lealtad de estos hacia el servicio, los resultados del estudio justifican la necesidad de una valoración global del servicio, la deter minación y desa rrollo de estrategias de gestión dirigidas principalmente para la mejora de la calidad en hombres y mayores de 56 años, el valor percibido en hombres y menores de 26 años y la satisfacción en los servicios deportivos que ofertan actividades organizadas, cuyo fin es conseguir la fidelidad de los clientes y atraer a consumidores potenciales.

\section{Referencias.}

Álvarez, J., González, E., Río-Rama, D., de la Cruz, M., \& Durán, A. (2019). Quality in Customer Service and Its Relationship with Satisfaction: An Innovation and Competitiveness Tool in Sport and Health Centers. International Journal of Environmental Research and Public $H$ ealth, 16(20), 39-42.

Aparicio, P., Elassi, A., \& Triadó, X. M. (2018). La satisfacción de los usuarios de actividades dirigidas de los Centros deportivos municipales de Barcelona. SPORT TKRevista EuroAmericana DeCiencias Del Deporte, 7(2), 27-34.
Barshan, G., Elahi,A. \& \& Aghæei, N. (2017). Impact of service quality on satiffaction, loyalty and intention to revisit of sport customers: The case study of swimming pools in Alborz Province. International Review of Management and Marketing, 7(2), 334-339.

Berber, U., \& Mollaodullar1, H. (2020).The effect of service quality on satisfaction of athletes participating in sport programmes. European Journal of Physical Education and Sport Science, 6(1), 1-11.

Bernal-García, A., Garcíafernández, J., Grao-Cruces, A., Teva Villén, R., \& Nuviala, A. (2018). Intenciones de comportamiento de los usuarios de servicios deportivosen función de las variables sociodemográficas, Revista Mexicana dePsicología, 35(1), 63-71.

Boksberger, P. E., \& Melsen, L. (2011). Perceived value: a critical examination of definitions, conceptsand measures for the service industry. Journal of services marketing, 25(3), 229-240.

Brady, M., Knight, G. A., Cronin, J., Hult, G., \& Keillor, B. (2005). Removing the contextual lens: A multinational, multi-setting comparison of service evaluation models. Journal of Retailing, 81(3), 215-230.

Calabuig, F., Burillo, P., Crespo, J., Mundina, J. J., \& Gallardo, L. (2010). Satisfacción, calidad y valor percibido en espectadores de atletismo. Revisa Internacional de Medicina y Ciencias de la Actividad Físca y de Deportel International Journal of Mediane and Science of Physical Activity and Sport, 10(40), 577-593.

Calabuig, F., Quintanilla, I., \& Mundina, J. (2008). Lacalidad percibida de los servicios deportivos: diferencias según la instalación, género, edad y tipo de usuario de servicios náuticos. Revista internacional de ciencias del deporte, $10(4), 25-43$.

Calesco, V. A., \& Both, J. (2020). Q ualidade dos serviç̧os prestados pela academias de ginástica (Calidad del servicio prestado por los gimnasios). Retos: NuevasTendencias en Educación Físca, Deportesy Recreación, 39, 18-23.

Campos, F., González, S., González, D., \& Martins, F. (2019). Benefits of 8-week fitness programs in health and fitness parameters. Retos: Nuevas Tendendas en Educación Físca, Deportesy Recreación, 35, 224-228.

Castãeda, C., Campos, M. C., \& Castillo, Ó. (2016). Physical activity and self-rated health in university students. Revista dela Facultad deM ediana, 64(2), 277-284. Castillo,A., O netti,W., \& Chinchilla, J. L. (2019). Perceived Quality in Sports Centers in Southern Spain: A Case Study. Sustainability, 11(14), 39-83.

Chiu, W., Won, D., \& Bæ, J. S. (2019). Customer value cocreation behaviour infitnesscenters: how doesitinfluence customers' value, satisfaction, and repatronage 
intention? M anaging Sport and Leisure, 24(1-3), 32-44.

Días, C., Ferreira, A., Romao-Pereira, A., \& Fonseca A. M., (2019). Examining the relationship between perceived service quality, satisfaction, and renewal intention in Portuguese fitness centers. Revista de Psicología del deporte, 28(2), 49-58.

Elasri, A., Triadó, X. M., \& Aparicio, P. (2014). La satisfacción de los clientes de los centros deportivos municipa les de Barcelona. Apuntes de Educación Física y Deportes, 199, 109-117.

García, F. G. , Alguacil, M., \& M olina, N. M. (2020). Predicción de la lealtad de los usuarios de centros deportivos privados. Variables de gestión y bienestar subjetivo (Prediction of the loyalty of costumers of private sports centers. Management variables and subjective wellbeing). Retos nuevas tendencias en educación física, deportey recreación, 38, 16-19.

García, C. S., Carcelén, C. G., Sánchez, G. L., \& Suárez, A. D. (2017). Satiffacción de clientes externos. Estudio de caso de una piscina cubierta. SPORT TK-Revista EuroAmericana de Ciencias del Deporte, 6(2), 81-88.

García, N. M. (2016). Evaluación de la calidad de servicio de centros deportivos privados. Influencia de la gestión en el bienestar delosusuarios. TesisD octora. U niversitat de València.

García, N. M ., \& García, R. J. G. (2018). Calidad percibida y satisfacción de los usuarios de unas escuelas náuticas. Journal of Sports Economics\& Management, 8(1), 37-62.

García, N. M., Gómez, J. M., \&Tafalla, A. G. (2019). Perfil del usuario de centros deportivos privados, según género, edad y nivel de antigüedad. SPORT TK-Revista EuroAmericana de Ciencias del Deporte, 8(1) 23-28.

García-Fernández, J., Bernal-García, A., Fernández-Gavira, J., \&Vélez-Colón, L. (2014).Analysis of existing literaure on management and marketing of the fitness centre industry. South African Journal for Research in Sport Physical Education and Recreation, 36(3), 75-91.

García-Fernández, J., Gálvez-Ruíz, P., Pitts, B., Vélez-CoIón, L., \& Bernal-García,A. (2018). Consumer behaviour and sport services: an examination of fitness center loyalty. International Journal of Sport M anagement and M arketing, 18(1-2), 8-23.

GarcíaFernández, J., Gavira, J. F., \& García, A. B. (2014). La percepción de calidad y fidelidad en clientes de centrosdefitnesslow cost. Suma psicológica, 21(2), 123-130.

GarcíaFernández, J., Grimaldi, M. , Gómez, R., \& Bernal, A. (2016). Calidad y valor en centros fitness low-cost: diferencia según característica de los clientes. Revista Intercontinental de Gestion D esportiva, 6(1), 63-72.

García-Pascual, F., Silla-M erchán, A., M undina, J., \&
EscamillaFajardo, P. (2016). The effect of management variables on subjective well-being of sports center users. Journal of Sports Economics\& M anagement, 6(2), 99111.

Grijalva, M., Cevallos, D. , \& Jiménez, M. (2020). Anáisis del perfil de usuario como herramienta parala disminución de abandonos en centros deportivos. mktDESCUBRE, 1(3), 107-117.

Haro, M. (2018). Servicios deportivos femeninos: compa ración entre servicios mixtos y exclusivos juicios de va lor e intenciones futuras. Tesis Doctoral. Universidad Pablo de O lavide.

Haro, M., Pérez-O rdás, R., Grao-Cruces, A., Nuviala, R., $\&$ Nuviala, A. (2018b). Female users of unisex fitness centers and of fitness centers exclusive for women: satisfaction. International Journal of Sports Marketing and Sponsorship, 19(4), 384-395.

Haro, M., Pérez-O rdás, R., Leyton, M., Caamaño, A., \& Nuviala, A. (2018a). Grupos de usuarias del mercado deportivo femenino. Retos: nuevas tendencias en educación física, deportey recreación, (34), 123-127.

IHRSA. The 2019 IHRSA Global Report; International Health; Racquet \& Sportsclub Association: Boston, MA, USA, 2019.

Kamolrat, P., de Lad, T., Mitas, O., \& de Rooij, P. (2019). Calidad de servicio, experiencia de servicio y satisfacción del cliente: el caso de un gimnasio en Breda, Páses Bajos. Revisa deEconomía y Estrategia de Gestión, 6(2), 123140.

León, J., García, J., \& Burillo, P. (2020). Service Perceptions in Fitness Centers: IPA Approach by Gender and Age. International Journal of Environmental Research and Public Health, 17(8), 28-44.

Molina, N. (2016). Evaluación de la calidad de servicio de centros deportivos privados. Influencia de la gestión en el bienestar de los usuarios. Tesis Doctoral. Valencia: PUV.

M olina-García, N. \& \& González-García, R. (2018). Perceived quality and users' satisfaction in sailing schools. Journal of Sports Economics \& Management, 8(1), 37-62.

Monroy, M. (2019). Factores de la satisfacción del cliente: perspectivas en restaurantes de comida rápida. Sinapsis, 11(1), 70 - 87.

Montesinos, E., Vegara, J. M., Morales, V., López, J. M., López, F., \& Angosto, S. (2018). Perceived quality, perceived value, satisfaction and future intentions in participants in swimming crossings. Journal of Physical Education and Sport, 18(1), 1316-1322.

Moreno, F. C., Prado-Gascó, V., Hervás, J. C., Núñez-Pomar, J. , \& Sanz, V.A. (2015). Spectator emotions: Effects on quality, satisfaction, value, and future 
intentions. Journal of BusinessResearch, 68(7), 1445-1449.

Murray, D., \& Howat, G. (2002). The relationships among service quality, value, satiffaction, and future intentions of customers at an Australian sports and leisure center. Sport Management Review, 5(1), 25-43.

Nicolás-López, J., \& Escaravajal-Rodríguez, J. (2020). Satisfacción con los servicios deportivos del Campus Universitario de Espinardo. SPORT TK-Revista EuroAmericana DeCienciasD el Deporte, 9(1), 105-112.

Nuviala, A., Grao-Cruces, A., Fernández-O zcorta, E., \& Nuviala, R. (2015). Association between Sports Service Q uality, Value and User Satisfaction in Spain. Universtas Psychologica, 14(2), 589-598.

Nuviala, A., Grao-Cruces, A., Teva, R., Pérez-Turpin, J.A., Pérez-O rdás, R., \& Tamayo, J. (2013). Duration of membership at sports centres and reasons for quitting. Perceptual and Motor Skills 117 (3), 733-741.

Nuviala, A., Pérez-O rdás, R., O suna, M., Grao-Cruces, A., Nuviala, R., \& Jurado, J. (2012). Calidad, satisfacción y valor percibido de los usuarios de un servicio deportivo público. Movimento, 18(4), 11-32.

Nuviala,A.,Tamayo-Fajardo, J.A., Gómez, C. R.A., Nuviala, R., \& Torres, J. M. D. (2017). Adaptación y validación del cuestionario Epod 2.1 a usuarios libres de centros deportivos Adaptation and validation of the Epod 2.1 questionnaire in free customers of sport centers. Retos. NuevasTendencias en Educación Física, Deporte y Recreación, 31, 40-45.

Nuviala, R., Aznar-Cebamanos, M., Nuviala, A., \& GraoCruces, $A$. (2020). Cultura organizativa del técnico deportivo y su relación con la Satisfacción y Valor percibido de usuarios de actividades deportivas extraescolares. Retos nuevastendenciasen educación físca, deportey recreación, 38, 606-612.

Ortega, J. I. , Lourenço, F, M. ., González-Villora, S., \& Campos, F. (2021). Estudio sobre la calidad percibida de los usuarios de fitness: a partir de sus características y preferencias según sexo y edad. Retos. nuevas tendencias en educación física, deportey recreación. 39, 477-482.

Pérez-O rdás, R., Aznar Cebamanos, M., Nuviala, R., \& Nuviala, A. (2019). Evaluation of extracurricular sports activities as an educational element for sustainable development in educational institutions. Sustainability, 11(12), 34-74.

Prado-Gascó, V., Calabuig, F., Crespo, J., \& Núñez-Pomar, J. (2018). Satisfaction of users of aquatic activities: Free swimming vs. guided activities. Journal of Physical Education and Sport, 18(3), 1427-1434.

Quintana, M.B., Molina, B., \& Molina, A. J. (2010). El turismo mayor de edad en Michoacán. Red Internacional de
Investigadoresen Competitividad, 4(1). 1216-1228.

Ramírez, C., Lorenzo, A. \& L López, C. (2017). Revisión de los instrumentos de medidadela calidad en losservicios deportivos. Journal Kronos, 16(1).

Ros-Castello, P., Calabuig, F., \& Gómez, A.M. (2018). Perfil del usuario de la fundación deportiva municipal de valencia y sus hábitos de práctica deportiva. Sport TK, 8(1), 17-22.

Salvador Ferrer, C. M. (2007). Diferencias de género en la percepción de la calidad del servicio. Un estudio exploratorio. Boletín de Psicología, 89, 29-45.

Solanellas, F., \& M acías, L. F. (2019). Comparative analysis on the perception of the quality of the municipal sports services of Baix Llobregat. Revista de Psicología del Deporte, 28(1), 105-114.

Solís-Radilla, M., Hernández-Lobato, L., \& VillagómezMéndez, J. (2016). El valor percibido del destino turístico en relación con el perfil del turistaen Acapulco, Guerrero-M éxico. Investigación administrativa, 45(118), 0-0. Theodorakis, N. D., Howat, G., Ko,Y. J. , \& Avourdiadou, S. (2014). A comparison of service evaluation models in the context of sport and fitness centres in Greece. Managing Leisure, 19(1), 18-35.

Vergara, J., Quesada, V., \& Maza, F. (2017). Efecto de los aspectos demográficos en la valoración de la calidad del servicio. Una revisión. Revisa U.D.C.A Actualidad \& Divulgación Científica, 20(2), 457-467.

Yildiz, S. M. . \& Duyan, M. (2019). The Relationship among service quality, customer satisfaction, and customer loyalty: An empirica investigation of sports and physical activity sector. Pamukkale Journal of Sport Sciences 10(1), 17-30.

Zamorano, S., \& García, J. (2018). The importance-performance analysis according to gender and membership length: the case of fitness centers. Materiales para la H istoria del $D$ eporte, 16, 24-35.

Zopiatis, A., Theocharous, A. L., Constanti, P., \&Tjiapouras, L. (2017). Quality, satisfaction and customers' future intention: The case of hotels' fitness centers in Cyprus. Journal of Quality Assurance in H ospitality \& Tourism, 18(1), $1-24$.

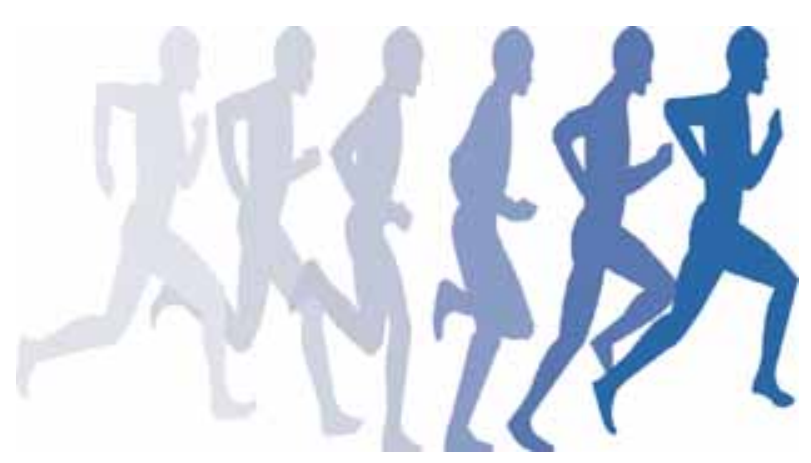

